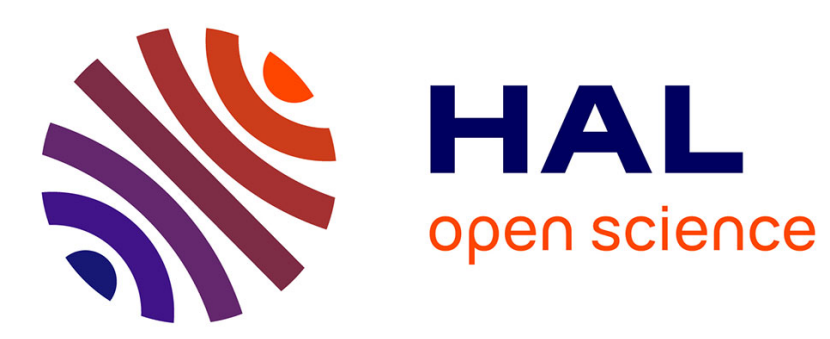

\title{
Graphical simulation of the dynamic evolution of the software architectures specified in $\mathrm{Z}$
}

\author{
Riadh Ben Halima, Mohamed Jmaiel, Khalil Drira
}

\section{To cite this version:}

Riadh Ben Halima, Mohamed Jmaiel, Khalil Drira. Graphical simulation of the dynamic evolution of the software architectures specified in Z. 8th International Workshop on Principles of Software Evolution, Sep 2005, Lisbon, Portugal. pp.4. hal-00438857

\section{HAL Id: hal-00438857 https://hal.science/hal-00438857}

Submitted on 4 Dec 2009

HAL is a multi-disciplinary open access archive for the deposit and dissemination of scientific research documents, whether they are published or not. The documents may come from teaching and research institutions in France or abroad, or from public or private research centers.
L'archive ouverte pluridisciplinaire HAL, est destinée au dépôt et à la diffusion de documents scientifiques de niveau recherche, publiés ou non, émanant des établissements d'enseignement et de recherche français ou étrangers, des laboratoires publics ou privés. 


\section{Graphical simulation of the dynamic evolution of the software architectures specified in $\mathrm{Z}$}

\author{
Riadh Ben Halima, Mohamed Jmaiel \\ University of Sfax \\ National School of Engineers \\ B.P.W, 3038 Sfax, Tunisia \\ E-mail: \{Riadh.BenHalima, Mohamed.Jmaiel\}@enis.rnu.tn
}

\author{
Khalil Drira \\ LAAS-CNRS \\ 7 avenue de Colonel Roche \\ 31007 Toulouse Cedex 4, France \\ E-mail : Khalil@laas.fr
}

\begin{abstract}
This paper provides a graphical simulator that enables to apprehend the dynamic of components-based software architectures based on their formal specification. The simulator initially accepts (as an input) an already validated $Z$ specification using the Z/EVES tool. Then, it generates graphical entities, according to the UML notation, representing software's components and their connectors. Thereafter, the user may generate architecture instances by adding components and connections between them. Architecture instances can be updated by destroying components/connections or by modifying their interconnections. The user actions are checked through the formal specification of the architectural style.
\end{abstract}

\section{Introduction}

The architecture of a software system is its "style and method of design and construction" [4]. A software system with dynamic architecture are adaptable applications whose architecture evolves during their execution. The evolution of a dynamic architecture results from the addition or the removal of components and connections between them, and from the modification of interconnections as described in $[5,7]$. Designing, this kind of applications is considered as a complex task which requires a rigourous specification of the corresponding architecture and its evolution.

In this work, we use formal methods for describing dynamic software architectures. We employ the $\mathrm{Z}$ notation [9] to specify architectural styles as well as configuration operations. The use of the formal approach allows us to rigourously reason about architectural properties and to prove that the architectural style is preserved during the application execution. In order to consolidate this formal approach, we design and implement a graphical tool that en- ables to apprehend the formal specification (written in Z) of an architecture. This tool parses $\mathrm{Z}$ specifications and allows to identify the elements of its architectural style as well as the pre- and the post-conditions associated to a reconfiguration operation. Moreover, it permits to generate diagrammatically architecture instances, using UML 2.0 notation [3], which respect the specified architectural style. Also, we integrate our simulator with the Z/EVES tool [8] which allows to edit, to check and to prove $\mathrm{Z}$ specification. Across its graphic interface, the simulator allows to the designer, who does not necessarily master formal specifications, to understand the behaviour of the system to build.

This paper is organized as follows. Section 2 gives a survey on the related work. Section 3 introduces the approach of the dynamic architectures description using Z. Section 4 presents the features and the design of our simulator. The last section concludes the paper.

\section{Related works}

Currently, the Unified Modelling Language (UML) is accepted as a standard for designing software applications. Its set of notations helps system designers to represent their solutions in a way that is expressive and yet easy to understand. However, UML is generally criticized for the lack of means for verifying and validating the designed models. The advantage of our approach appears in its capacity to produce a formal model presented to the user according to the UML visual notations during the simulation process. So, on one hand, contrary to an approach using only the formal, we suppose that the user of our environment does not need to be an expert in formal methods. On the other hand, we do not reduce the rigourous capacity of our model by limiting us to the informal side, using only the UML notations.

Several research works choose the use of formal methods for specifying dynamic software architectures. Among 
these works, we mention the CHAM [10] approach which is based on grammars and rewriting rules. This approach uses reactions and evolutions to formalize, respectively, architectural styles and reconfigurations. However, We note the lack of tools for validating or simulating specifications developed according to this approach. Other approaches, like LEDA [2] and Wright [1] make use of process algebra to specify dynamic software architectures. Wright presents the structure of an architecture as a graph and specifies the system behaviour and reconfigurations using a variant of CSP. LEDA uses the $\pi$-calculus to describe dynamic architectures. It supports prototype execution using a $\pi$-calculus analysis tool : the Mobility Workbench. Another widely recognized approach is RAPIDE [6], in which a software architecture consists of a set of module interfaces interconnected according to a set of connection rules and communication constraints. These prominent approaches, although they propose rigorous solutions for designing software architectures, they lack tools for simulation and animation of software architectures. RAPIDE is one of the few formalisms which enable the animation of a program behaviour in term of causal ordering of events.

On the other hand, there are several tools which tried to animate $\mathrm{Z}$ specifications, namely, Jaza (Just Another Z Animator), PiZA (A $Z$ Animator which is implemented in Prolog) and ZANS (Z ANimator System). Firstly, Jaza is used to evaluate the schemas of a $Z$ specification. However, it presents some drawbacks. Among his limits, we can appoint : the absence of the treatment of all the $\mathrm{Z}$ language constituents (e.g. generic definitions and axiomatic declarations), the impossibility of declaring the functions or relations infixed, prefixed and postfixed. Then, PiZA allows the conversion of restricted parts of a $\mathrm{Z}$ specification into Prolog and their execution. It is hard to use because it requires the installation of other software and contains at least two translation stages. Finally, ZANS permits the evaluation of expressions and predicates and the execution of operation schemas. It allows animating finite subsets of $Z$ specifications. Nevertheless, a formal specification animator executes and interprets traces on a specification. Thus, animation can only show the presence of errors, never their absence. Whereas, the visual simulation displays the specification lacks and deficits and gives more chance to fix errors.

\section{Specification of dynamic architectures}

Our work is based on the approach [5], which relies on the integration of graph grammars within the $\mathrm{Z}$ notation to describe the static and dynamic aspects of software architectures. Its advantage is that it is expressive enough to deal with the static aspects (component types, connection types and architectural properties) as well as the dynamic aspects (reconfiguration operations) of an architecture. This approach aims, mainly, at guaranteeing the preservation of architectural properties during the system evolution. According to this approach, the architectural style of a software system is described using a $\mathrm{Z}$ schema as follows :

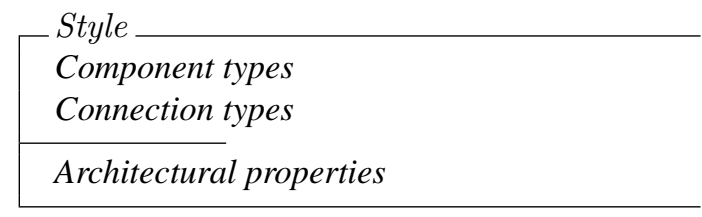

Schema 1: Architectural style

To give more details on our approach, we present in this section a simple example. The Patient Monitoring System $(P M S)$, which was used to illustrate work of [7]. It is a software system which let the nurses (of type NURSE) controlling their remote patients in a private clinic. A controller (of type BED_MONITOR) is attached to the bed of the patient.

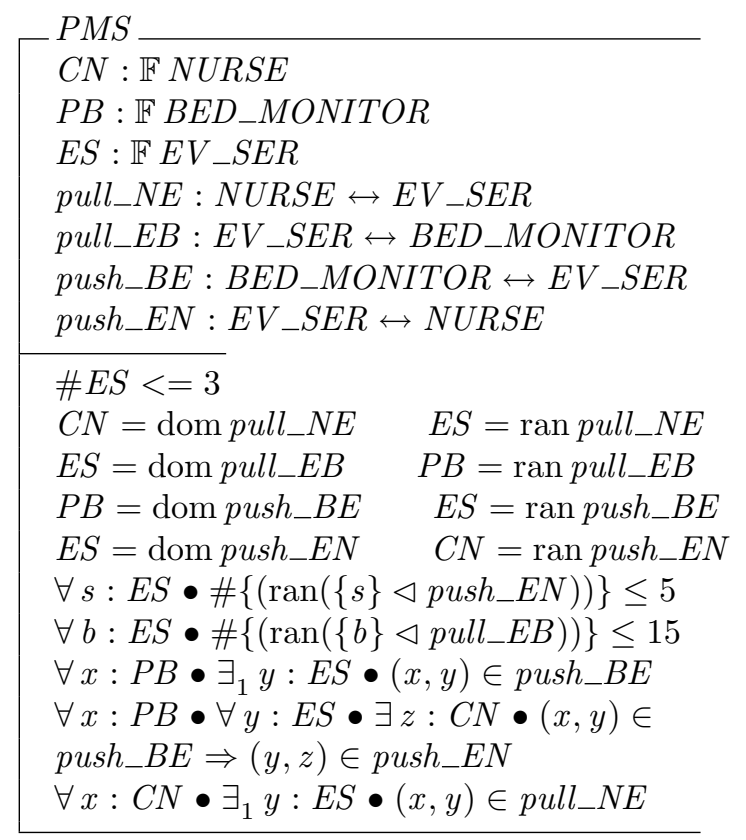

Schema 2: PMS schema

For each service of the private clinic (pediatric, cardiology, maternity...), we associate an event service (of type $E V \_S E R V$ ) to manage the communications between nurses and bed monitors (when necessary). The architectural style is represented by schema 2 .

In the first part this schema, we develop a declaration part which specifies component types as well as connection types being able to exist between them. In this specification, $C N, P B$ and $E S$ represents respectively the nurses, the bed monitors and the event services. This part integrates also relations representing the communication links 
between the components. In addition to the architectural style constraints, an application can have specific properties which must be satisfied during the evolution of its architecture. We will take some properties of the PMS system, which are formulated in the second part of schema 2, such:

- The system must contain a maximum of 3 services.

- A service contains a maximum of 5 nurses and 15 patients.

- A nurse must be connected to only one service.

According to the approach presented in [5], the dynamic part of an architecture is described using $\Delta$ (Delta) $\mathrm{Z}$ schemas (cf Schema 3). Indeed, each $\Delta$ schema represents a reconfiguration operation.

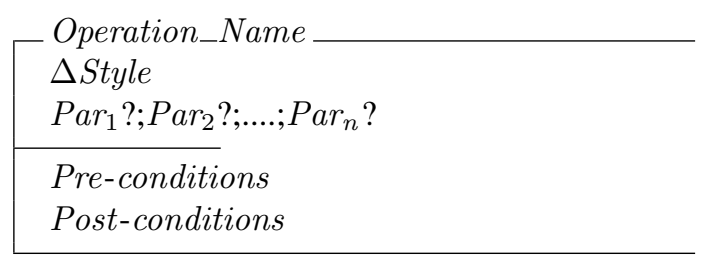

\section{Schema 3: A Delta schema}

Where $\mathrm{Par}_{i}$ ? denotes an input of the operation. The PMS specification presents different operations of reconfiguration that make possible system evolution. The reconfiguration is then performed if the functional and structural constraints are satisfied. For example, the Insertion of an event-service operation (cf Schema 4) inserts an instance of a component of type $E V \_S E R$ provided that the system does not already contain three event services according to the first predicate in the PMS schema.

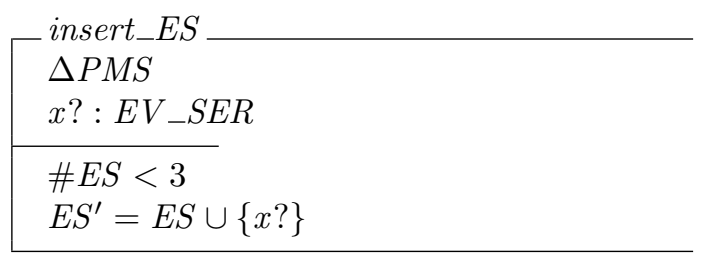

\section{Schema 4: Insertion of an event-service}

We used the Z/EVES tool [8] to edit and check the syntax of the PMS specification as well as to prove that the specified reconfiguration operation preserve the architectural style. Indeed, if we start from a configuration which satisfies the architectural style and we apply an operation of reconfiguration to the latter, we obtain a new configuration which also satisfies the architectural style. Formally and in the case of the PMS specification, this is translated in the following theorem:

$$
\begin{aligned}
& \forall p m s \in P M S, \forall o p \in O p \_P M S, p m s \wedge o p \Rightarrow p m s^{\prime} \\
& \wedge\left(p m s^{\prime} \text { satisfy the PMS pre-conditions }\right)
\end{aligned}
$$

With, $O p \_P M S=i n s e r t \_E S$, insert_CN, insert_PB, supp_PB, supp_CN, supp_ES;

With Z/EVES, the demonstration of this theorem corresponds to demonstrate six sub-theorems where each considers an operation from the set $O p \_P M S$. For example, for the insert_ES operation, it is necessary to demonstrate the following theorem:

$$
\begin{aligned}
& \text { theorem Verif_insert_ES } \\
& P M S \wedge \text { insert_ES } \Rightarrow P M S^{\prime} \wedge\left(P M S^{\prime} \Rightarrow \text { pre } P M S\right)
\end{aligned}
$$

This theorem, proved by Z/EVES, shows that the initial architectural style is preserved. The following section debates the visual simulation of a $\mathrm{Z}$ specification.

\section{The simulator : presentation and features}

The simulator is a generic application which simulates the functioning mode of component-based applications. It represents components and connections using UML 2.0 conventions. Moreover, it provides the possibility of adding and/or removing components and connections. Each reconfiguration action (adding/removing) proceeds while respecting its description within the $\mathrm{Z}$ notation, according to the approach presented in Section 3. The main interface of the simulator (figure 1) contains menus, a toolbox and a drawing panel.

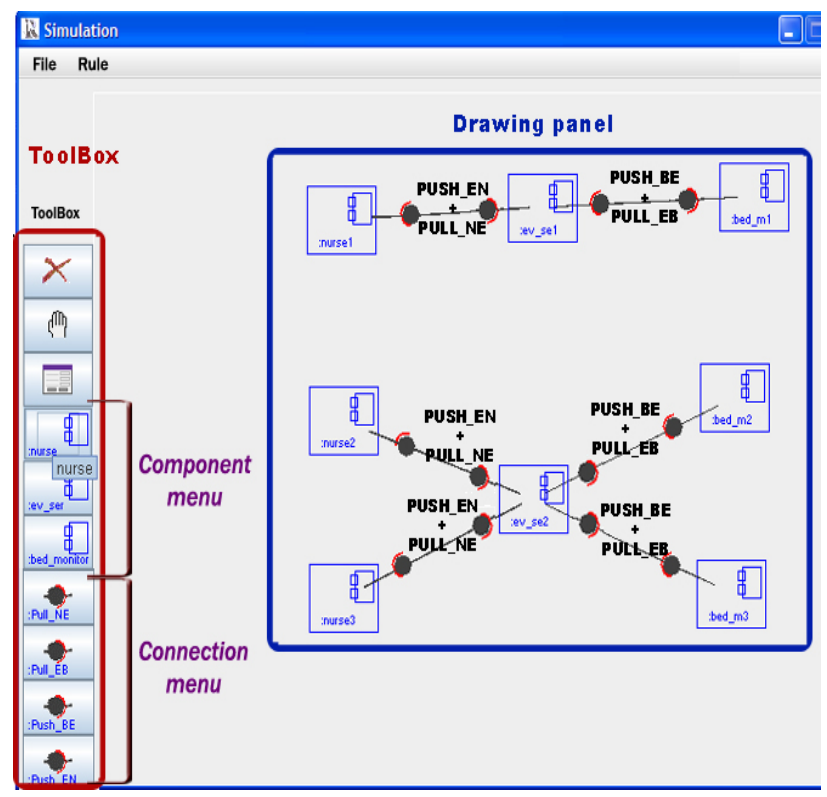

Figure 1. The simulator GUI

We group in figure 2 the different operating scenarios of our simulation environment. Its use proceeds according to following phases :

1. The start up of the simulator.

2. The user commands to the simulator to read the specification (with the $\mathrm{Z}$ notation) to simulate. 
3. The simulator parses the specification in order to extract the architectural style (the types of components and connections, as well as the constraints) and to list the reconfiguration operations.

4. The simulator prepares the toolbox (for each component or connection type corresponds a button).

5. The simulation of reconfigurations: the user press on a button in the toolbox (which represents a component type or a connection type) and clicks on the drawing panel in order to represent graphically the component chosen according to the UML 2.0 notation.

6. The simulator checks the compatibility of each reconfiguration with the architectural style. If it generates an architecture instance which respects the style, it executes the reconfiguration, otherwise it breaks it.

7. If during simulation the user detects a case which does not comply with the requirements, then we are brought to the initial $\mathrm{Z}$ specification. To take into account of the new synthesized property, we use the command Update of the menu Rule. Thus we put the new changes into practice.

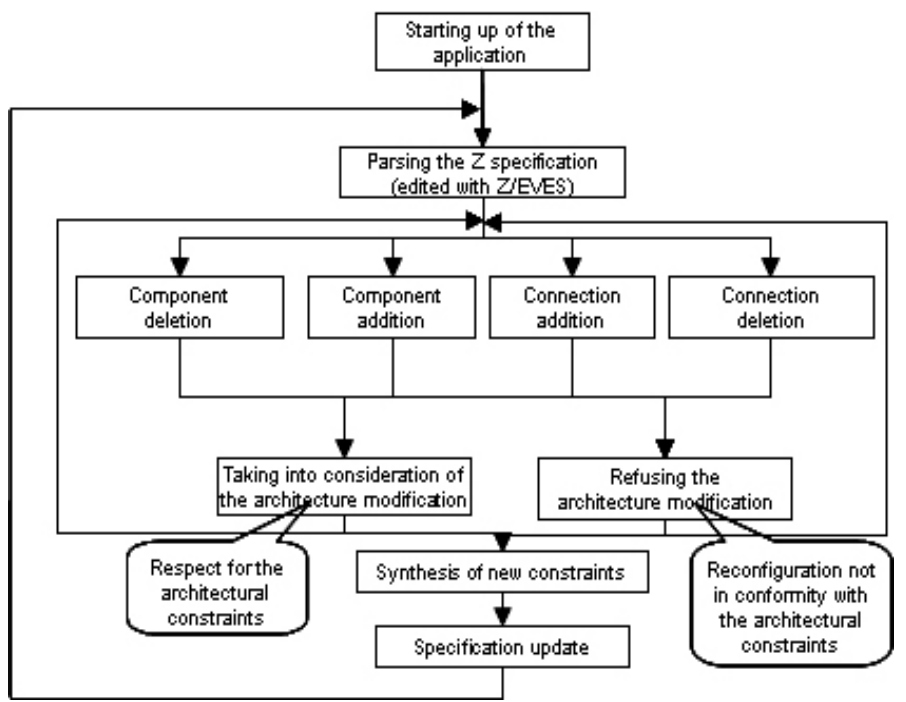

Figure 2. Functional scenario

Our simulator examines and translates the architectural properties into a set of constraints to be respected by the user during the simulation of a reconfiguration. If an operation does not obey the constraints, the simulator prohibits it while showing an error message. For example, when we begin with a configuration that contains three events services and we want to add the fourth, we creates an inconsistent state (because \# ES $<=3$ ). Therefore, our simulator ignores this reconfiguration. Also, we cannot bind two components as long as there is no connection specified in the architectural style between them. For example, it is impossible to bind a nurse with a bed monitor because no relation between them is specified. Additionally, we can update the specification whenever we want to satisfy user's need. An example in the case of PMS: Due to the small number of patients, the clinic manager wants to reduce the expenses. He wants to limit the nurses's number to twenty. To allow the taking into account of the new rule (in the $\mathrm{Z}$ specification), we add the following line to the architectural properties of PMS : |\# CN $<20$. To forbid the simulation of such configuration, the simulation must reload the modified specification with the update command. After that, the simulator prohibits any configuration to simulate with a nurses number superior to twenty.

3.

\section{Conclusion}

Our purpose in this paper is to provide to the designers a visual tool that allows to conceive UML2.0 diagram of a system according to his formal specification with the $\mathrm{Z}$ notation. The interest of this environment is to enable designers, who are not familiar with formal techniques, to better understand the architectural properties of the system formally specified with $\mathrm{Z}$. The graphic visualization of an erroneous configuration helps to identify the specification defects and to rectify them.

In perspective, we intend to take into account the behaviour of components. For that goal, we envisage the integration of a formal language which supports the description of processes such as process algebra.

\section{References}

[1] R. Allen, R. Douence, and D. Garlan. Specifying and analyzing dynamic software architectures. Lecture Notes in Computer Science. 1998.

[2] C. Canal, E. Pimentel, and J. M. Troya. Specification and refinement of dynamic software architectures. In In Software Architecture, pages 107-126. Kluwer Academic Publishing, February 1999.

[3] O. M. Group. Uml superstructure 2.0 - draft adopted specification. 2003.

[4] B. Hayes-Roth, K. Pfleger, P. Lalanda, P. Morignot, and M. Balabanovic. A domain-specific software architecture for adaptive intelligent systems. IEEE Trans. Softw. Eng., 21(4):288-301, 1995.

[5] I. Loulou, A. H. Kacem, M. Jmaiel, and K. Drira. Toward a unified graph-based framework for dynamic componentbased architectures description in z. In ACS/IEEE International Conference on Pervasive Services ICPS'04, 2004.

[6] D. C. Luckham, J. L. Kenney, L. M. Augustin, J. Vera, D. Bryan, and W. Mann. Specification and analysis of system architecture using rapide. In IEEE Trans. on Software Engineering, volume 21, pages 336-355, 1995.

[7] D. L. Métayer. Describing software architecture styles using graph grammars. IEEE Transactions on Software Engineering, 24(7):521-553, July 1998.

[8] ORA. Z/eves. http://www.ora.on.ca/Z-eves.

[9] J. Spivey. The Z Notation : a Reference Manual, Series in Computer Science. Prentice-Hall, 1992.

[10] J. Vera, L. Perrochon, and D. C. Luckham. Event-based execution architectures for dynamic software systems. In the Working IFIP Conf. on Software Architecture, pages 22-24, 1999. 